\title{
SPIN REPRESENTATION OF INVERSIONS $\dagger$
}

\author{
A. H. TAUB
}

1. Introduction. The spin representation of the (real or complex) orthogonal group in a euclidean space $E_{n}$ of $n=2 \nu$ or $2 \nu+1$ dimensions may be obtained by setting up a correspondence between the lines through a fixed point (the origin) in $E_{n}$ and a linear family of involutions in a projective space $P_{2^{\nu}-1}$ of $2^{\nu}-1$ dimensions. $\neq$

Analytically the correspondence is set up by finding $n 2^{\nu}$-dimensional matrices $\gamma^{i}$ which form a basis for the linear family and which satisfy the relation

$$
\frac{1}{2}\left(\gamma^{i} \gamma^{j}+\gamma^{j} \gamma^{i}\right)=\delta^{i j} \cdot 1, \quad i, j=1, \cdots, n .
$$

The existence and properties of these matrices and the properties of the algebra for which they generate a basis have been discussed in detail by $\mathrm{R}$. Brauer and $\mathrm{H}$. Weyl $\S$ and others. The main result which will be used here is that to every proper orthogonal transformation in $E_{n}$ there corresponds a unique collineation (a pencil of $2^{\nu}$-dimensional matrices) in $P_{k-1},\left(k=2^{v}\right)$, which leaves the linear family invariant, and conversely. In case $n$ is even, the image in $P_{k-1}$ of an improper orthogonal transformation in $E_{n}$ is also a collineation, but if $n$ is odd and $\nu>1$, the image of such a transformation in $E_{n}$ is a correlation in $P_{k-1}$. In case $n$ is odd and $\nu=1$, the image of such a transformation in $E_{n}$ is an antiprojectivity. The matrix representing the orthogonal transformation may be normalized to within a sign; that is, the representation is double-valued.

If $\gamma^{i}$ are solutions of (1), $\mathrm{a}_{j}^{i}$ the coefficients of an orthogonal transformation in $E_{n}$ (proper if $n$ is odd but arbitrary if $n$ is even), and $A$ the $2^{\nu}$-dimensional matrix in $P_{k-1}$ corresponding to $a_{j}{ }^{i}$, then they are related by the equations

$$
a_{j}^{i} \gamma^{j}=A \gamma^{i} A^{-1}
$$

or

$$
\gamma^{i}=a_{j}^{i} A^{-1} \gamma^{i} A .
$$

$\dagger$ Presented to the Society, September 9, 1937.

$\ddagger$ See O. Veblen and J. von Neumann, Geometry of Complex Domains, Princeton Mimeographed Notes, 1935-1936.

$\S \mathrm{R}$. Brauer and H. Weyl, Spinors in $n$ dimensions, American Journal of Mathematics, vol. 57 (1935), pp. 425-449. 
If we use preferred (cartesian) coordinates in $E_{n}$, then the quantities $\gamma^{i}, a_{j}{ }^{i}$, and $A$ are all constants.

In order to describe these results in a general coordinate system in $E_{n}$, we note the transformation properties of the $\gamma_{i}$. Under an arbitrary transformation of coordinates in $E_{n}$ the $n$ matrices $\gamma^{i}$ transform as a contravariant vector, whereas under an arbitrary transformation of coordinates in $P_{k-1}$ they transform as collineations. The term "frame of reference" will be used to denote a coordinate system in each of the spaces $E_{n}$ and $P_{k-1}$. We note that the frame of reference may be changed by changing coordinate systems in either or both of the spaces $E_{n}$ and $P_{k-1}$. Under an arbitrary change of reference frame the $\gamma^{i}$ have the transformation law

$$
\gamma^{i *}\left(x^{*}\right)=\frac{\partial x^{i *}}{\partial x^{i}} T^{-1}\left(x^{*}\right) \gamma^{i}\left(x\left(x^{*}\right)\right) T\left(x^{*}\right)
$$

In order to find the spin image of any transformation $x^{i *}=f^{i}(x)$ in $E_{n}$, we must solve equations (4) for the matrix $T(x)$ such that the matrix $\gamma^{i *}\left(x\left(x^{*}\right)\right)$ is a solution of the equations

$$
\frac{1}{2}\left(\gamma^{i *} \gamma^{j *}+\gamma^{j *} \gamma^{i *}\right)=g^{i j *}=g^{k l} \frac{\partial x^{i *}}{\partial x^{k}} \frac{\partial x^{j *}}{\partial x^{l}},
$$

and $g^{k l}$ is the value of the metric tensor $E_{n}$ in the general coordinate system described by the coordinates $x^{i}$. It is evident that if $T$ is the image of $x^{i *}=f^{i}(x)$, and $S$ is the image of $x^{* *_{i}}=g^{i}\left(x^{*}\right)$, then $T S$ is the image of $x^{* *_{i}}=g^{i}(f(x))$.

If an orthogonal transformation in $E_{n}$ is applied to a general coordinate system (for example, polar coordinates) in which the metric tensor is given by $g_{i j}(x)$, then in the new coordinate system $g_{i j}^{*}\left(x^{*}\right)=g_{i j}\left(x^{*}\right)$. Hence in this case $\gamma^{i *}\left(x^{*}\right)=\gamma^{i}\left(x^{*}\right)$ are a solution of (5), and equation (4) becomes

$$
\gamma^{i}\left(x^{*}\right)=\frac{\partial x^{i *}}{\partial x^{j}} T^{-1}\left(x^{*}\right) \gamma^{i}\left(x\left(x^{*}\right)\right) T\left(x^{*}\right) .
$$

It is evident that equation (6) reduces to (3) when we use cartesian coordinates in $E_{n}$. Moreover, in these coordinates the matrices $T$ are constant.

In another paper equation (6) will be used to investigate the spin representations of groups of motion of Riemannian spaces. In this paper we use equation (4) to obtain the spin representation of the inversion in the unit hypersphere in $E_{n}$, namely, the transformation

$$
I: \quad x^{i *}=x^{i} / r^{2},
$$


where the $x^{i}$ are cartesian coordinates and

$$
r^{2}=\delta_{i j} x^{i} x^{i}
$$

The summation convention is used throughout this note, and since we use cartesian coordinates, no distinction need be made between covariant and contravariant indices.

2. The image of $I$. From equation (7) it follows that

$$
\frac{\partial x^{i *}}{\partial x^{j}}=\frac{1}{r^{2}}\left(\delta_{j}^{i}-\frac{2 x^{i} x^{j}}{r^{2}}\right)
$$

and hence that

$$
g^{i j *}=\delta^{k l} \frac{\partial x^{i *}}{\partial x^{k}} \frac{\partial x^{j *}}{\partial x^{l}}=\frac{1}{r^{4}} \delta^{i j} .
$$

Therefore the matrices

$$
\gamma^{i *}=-\frac{1}{r^{2}} \gamma^{i}
$$

are solutions of (5) if $\gamma^{i}$ are solutions of (1). Equations (4) then become

$$
-\frac{1}{r^{2}} \gamma^{i}=\frac{1}{r^{2}}\left(\delta_{j}^{i}-\frac{2 x^{i} x^{j}}{r^{2}}\right) T^{-1} \gamma^{j} T .
$$

A solution of these equations is given by the matrix

$$
T=\frac{1}{r} x^{j} \gamma_{j}=\frac{x^{i *}}{r^{*}} \gamma_{i}
$$

as is readily verified by using equations (1). Hence the matrix $T$ given by equations (12) is the spin image of the inversion $I$ given by equations (7). From equation (1) it follows that

$$
T^{2}=1,
$$

as it must since $I$ is a transformation of period two. This condition together with (11) determines the matrix $T$ except for sign.

If the transformation $I$ is followed by the rotation

$$
x^{* * i}=a_{j}^{i} x^{* j}
$$

in $E_{n}$, we obtain the transformation

$$
x^{* * i}=a_{j}^{i} x^{j} / r^{2}
$$


in $E_{n}$. If we first apply (13) and then (7), we obtain the transformation

$$
x^{* * i}=\frac{x^{* i}}{r^{* 2}}=\frac{a_{j}^{i} x^{j}}{r^{2}} .
$$

Hence the transformation $I$ commutes with any orthogonal transformation in $E_{n}$. Therefore the direct product of the group of two elements, the identity and $I$, and the orthogonal group of $E_{n}$ forms a group $G$ in $E_{n}$. This group is a subgroup of the conformal group in $E_{n}$. The dilations and translations which are in the conformal group are not in this subgroup.

We next investigate the spin representation of the group $G$. If $n$ is even and $A$ is the image of an orthogonal transformation in $E_{n}$, and if $T$ is the image of $I$, then $A T(x)$ is the image of the transformation (14). This is the same transformation as $T\left(x^{*}\right) A$, where $x^{*}=a_{j}{ }^{i} x^{j}$. For

$$
A T A^{-1}=\frac{1}{r} A\left(x^{i} \gamma_{i}\right) A^{-1}=\frac{1}{r} x^{i} a_{\imath}{ }^{j} \gamma_{j}=\frac{x^{i *}}{r^{*}} \gamma_{i}=T\left(x^{*}\right)
$$

In case $n$ is odd, the above discussion gives a spin representation of that subgroup of $G$ composed of the direct product of the proper orthogonal transformations of $E_{n}$ and the group of two elements, the identity and $I$. By adjoining the correlation (or antiprojectivity if $\nu=1)$ in $P_{k-1}$, which corresponds to an improper orthogonal transformation in $E_{n}$, to the spin representation of this subgroup of $G$, we obtain the spin representation of $G$. Thus in both cases we obtain a spin representation of all the conformal transformations except dilations and translations. This is a collineation representation in the sense that if the $2^{v}$-dimensional matrix $P$ corresponds to a transformation of $G$, so does $\rho P$, where $\rho$ is an arbitrary constant different from zero.

3. Dirac equations and inversions. The results of the previous section will be applied to the study of the invariance properties of the Dirac equations under inversions. The Dirac equations may be written as

$$
\gamma^{j}\left(\frac{\partial}{\partial x^{j}}-\frac{e}{c} \frac{i}{h} \phi_{j}\right) \psi=\frac{m i}{h} \psi=\mu \psi,
$$

where $\phi_{j}$ is the four-dimensional vector potential of the external field, $e$ the charge on the electron, $m$ its mass, $c$ the velocity of light, and $h$ Planck's constant divided by $2 \pi$. Under the transformation of co- 
ordinates which is given by equations (7), equation (15) becomes

$$
\gamma^{j *}\left(\frac{\partial}{\partial x^{j *}}-\frac{e}{c} \frac{i}{h} \phi_{i}^{*}\right) \psi=\mu \psi,
$$

where

$$
\gamma^{j *}=\gamma^{i} \frac{\partial x^{* j}}{\partial x^{i}}, \quad \phi_{j}^{*}=\phi_{i} \frac{\partial x^{i}}{\partial x^{j *}}
$$

If we now let

$$
\psi=\frac{1}{r^{*}}\left(x^{* j} \gamma_{j}\right) \psi^{*}=T \psi^{*}
$$

equation (16) becomes

$$
\gamma^{j *}\left(\frac{\partial T}{\partial x^{j *}} \psi^{*}+T \frac{\partial \psi^{*}}{\partial x^{j *}}-T \frac{e}{c} \frac{i}{h} \phi_{j}^{*} \psi^{*}\right)=\mu T \psi^{*}
$$

or

$$
T \gamma^{j *} T\left(\frac{\partial}{\partial x^{j *}}-\frac{e}{c} \frac{i}{h} \phi_{j}^{*}+T \frac{\partial T}{\partial x^{j *}}\right) \psi^{*}=\mu \psi^{*} .
$$

In virtue of equations (11) this becomes

$$
-\frac{1}{r^{2}} \gamma^{j}\left(\frac{\partial}{\partial x^{j *}}-\frac{e}{c} \frac{i}{h} \phi_{j}^{*}+T \frac{\partial T}{\partial x^{j *}}\right) \psi^{*}=\mu \psi^{*} .
$$

But

$$
\begin{aligned}
\frac{\partial T}{\partial x^{i *}} & =\frac{1}{r^{*}} \gamma_{i}-\frac{1}{r^{* 3}} x^{* i}\left(x^{j *} \gamma_{j}\right), \\
T \frac{\partial T}{\partial x^{* i}} & =\frac{1}{r^{* 2}}\left[\left(x^{j *} \gamma_{j}\right) \gamma_{i}-x^{i *}\right]=\frac{1}{r^{* 2}}\left[x^{j *}\left(2 \delta_{i j}-\gamma_{i} \gamma_{j}\right)-x^{i *}\right] \\
& =\frac{1}{r^{* 2}}\left[x^{i *}-\gamma_{i}\left(x^{j *} \gamma_{j}\right)\right], \\
\gamma^{i} T \frac{\partial T}{\partial x^{i *}} & =\frac{1}{r^{* 2}}\left(\gamma^{i} x^{i *}-4 x^{i *} \gamma_{i}\right)=-\frac{3}{r^{2}}\left(x^{i *} \gamma_{i}\right) .
\end{aligned}
$$

Hence equation (17) becomes

$$
-\frac{1}{r^{2}} \gamma^{j}\left(\frac{\partial}{\partial x^{j *}}-\frac{3 x^{j *}}{r^{* 2}}-\frac{e}{c} \frac{i}{h} \phi_{j}^{*}\right) \psi^{*}=\mu \psi^{*} .
$$


If we now let $\psi^{*}=r^{* 3} \psi^{\prime}$, this equation becomes

$$
\gamma^{j}\left(\frac{\partial}{\partial x^{j *}}-\frac{e}{c} \frac{i}{h} \phi_{j}^{*}\right) \psi^{\prime}=-\mu r^{2} \psi^{\prime} .
$$

The factor $-r^{2}$ on the right-hand side of this equation shows that the Dirac equation for an electron is not invariant under inversions. However, if we set $\phi_{j}=0$ and $\mu=0$, then equation (15) is numerically invariant under inversions. This is done in the neutrino theory of light. Hence that theory has the same invariance properties as the Maxwell theory. Veblen $\dagger$ and Dirac $\ddagger$ have both shown that there is no nonsingular analog of the Dirac equation which is conformally invariant. The result given here shows how the invariance fails. We have given the detailed treatment of the behavior of the Dirac equation under the four-dimensional inversion; the three-dimensional inversion may be treated by restricting the range of indices in (7), (8), and (12) to $1,2,3$ and adding the equation $x^{4 *}=x^{4}$ to (7).

UNIVERSITY OF WASHINGTON

\section{NOTE ON DEGREE OF TRIGONOMETRIC AND POLYNOMIAL APPROXIMATION TO AN ANALYTIC FUNCTION $\$$}

\section{J. L. WALSH AND W. E. SEWELL}

1. Introduction. Well known results $\|$ relate the continuity properties of a real function $f(x)$ to the degree of approximation to $f(x)$ by trigonometric sums and by polynomials in $x$. In more recent years further results $\uparrow$ have related the continuity properties of a complex function $f(z)$ to the degree of approximation to $f(z)$ by polynomials in the complex variable $z$. The object of the present note is to obtain some new results lying on the border line of these two general fields of research.

To be more explicit, if $f(z)$ is analytic in the annulus $\rho>|z|>1 / \rho<1$, the degree of convergence on $|z|=1$ of the Laurent development of

$\dagger \mathrm{O}$. Veblen, $A$ conformal wave equation, Proceedings of the National Academy of Sciences, vol. 21 (1935), p. 484.

$\ddagger$ P. A. M. Dirac, Wave equations in conformal space, Annals of Mathematics, (2), vol. 37 (1936), p. 429.

$\S$ Presented to the Society, September 6, 1938.

|| Due especially to S. Bernstein, Jackson, Lebesgue, Montel, and de la Vallée Poussin.

I Due especially to J. Curtiss, Sewell, and Walsh. 